\title{
Correlação espacial da mortalidade perinatal com condições sociais, econômicas e demográficas: estudo ecológico
}

\author{
Spatial correlation of social, economic and \\ demographic conditions with perinatal mortality: an \\ ecological study
}

Michelle Thais Migoto', Rafael Pallisser de Oliveira², Luciano de Andrade³, Márcia Helena de Souza Freire ${ }^{4}$

\begin{abstract}
1. Enfermeira. Mestre em Enfermagem. Doutoranda no Programa de Pós-Graduação em Enfermagem na Universidade Federal do Paraná (UFPR). Curitiba - Paraná - Brasil. ORCID: https://orcid.org/00000002-8546-8694. E-mail: michellemigoto@gmail.com

2. Analista de Sistemas. Especialista em Business Intelligence e Business Analytics. Cursando Data Science e Big Data na Universidade Positivo. Curitiba - Paraná - Brasil. ORCID: https://orcid.org/0000-00026997-399X. E-mail: rafaelpallisser@gmail.com

3. Enfermeiro. Professor Adjunto no Departamento de Medicina na Universidade Estadual de Maringá. Maringá - Paraná - Brasil. ORCID: https://orcid.org/0000-0003-2077-1518. E-mail: luc.and1973@ gmail.com

4. Enfermeira. Doutora em Saúde Pública. Professora Adjunta no Departamento de Enfermagem da UFPR. Curitiba - Paraná - Brasil. ORCID: https://orcid.org/0000-0003-3941-3673. E-mail: marciahelenafreire@gmail.com
\end{abstract}

CONTATO: Michelle Thais Migoto | Endereço: Avenida Pref. Lothario Meissner. 632. Bloco Didático II. $3^{\circ}$ andar | Jardim Botânico | Curitiba | Paraná | Brasil | CEP 80210-170 | Telefone (41) 98760-0349 | E-mail: michellemigoto@gmail.com

COMO CITAR: Migoto TM, Oliveira RP, Andrade L, Freire MHS. Correlação espacial da mortalidade perinatal com condições sociais, econômicas e demográficas: estudo ecológico. R. Saúde Públ. Paraná. 2020 Jul;3(1):75-85. 
RESUMO Apesar da diminuição dos óbitos infantis, ainda se observa a necessidade de continuidade de redução, sobretudo da mortalidade perinatal, que se encontra distante dos resultados dos países desenvolvidos os quais abrangem um dígito. Objetivo: investigar a correlação espacial entre a mortalidade perinatal e os indicadores sociais, econômicos e demográficos dos municípios do Paraná. Método: estudo epidemiológico, tipo ecológico, com dados secundários. Utilizadas as variáveis de mortalidade, sociais, econômicas e demográficas, analisadas pelo Índice de Moran e pelo Indicador Espacial de Associação Local. Resultados: identificou-se autocorrelação espacial direta entre mortalidade perinatal $(0,638)$ com analfabetismo $(0,183)$, fecundidade entre 15 a 17 anos $(0,074)$ e Índice de Gini $(0,143)$ e, ainda, autocorrelação negativa para taxa de atividade $(-0,142)$, grau de urbanização $(-0,111)$ e Índice de Desenvolvimento Humano Municipal $(-0,276)$. Conclusão: a análise espacial permitiu confirmar a relação entre a mortalidade perinatal e as condições sociais, econômicas e demográficas, bem como, a identificação das regiões que necessitam de investimentos sociais e em saúde devido a maior vulnerabilidade a este tipo de óbito.

PALAVRAS-CHAVE: Mortalidade Perinatal. Saúde Materno-Infantil. Análise Espacial. Condições Sociais.

\begin{abstract}
Despite the decrease in infant deaths, there is still a need to reduce them, especially Perinatal Mortality, which is far from the results of developed countries, which are of one digit. Objective: to investigate a spatial correlation between Perinatal Mortality and social, economic and demographic indicators in the municipalities of the state of Paraná. Method: epidemiological, ecological study, with secondary data. Mortality, social, economic and demographic variables were used and analyzed through the Moran Index and the Local Association Spatial Indicator. Results: A direct spatial autocorrelation was identified between Perinatal Mortality (0.638) and Illiteracy (0.183), Fertility between 15 and 17 years (0.074) and Gini Index (0.143), and also negative autocorrelation for Activity Rate (-0.142), Level of Urbanization (-0.111), and Municipal Human Development Index (-0.276). Conclusion: The spatial analysis allowed the confirmation of a relationship between Perinatal Mortality and social, economic and demographic conditions, and the identification of regions that require social and health investments because they are more vulnerable to this type of death.
\end{abstract}

KEYWORDS: Perinatal Mortality. Maternal and Child Health. Spatial Analysis. Social Conditions.

\title{
INTRODUÇÃO
}

A

mortalidade perinatal é um indicador que permite avaliar as condições de saúde da população materna e infantil, pois estima o risco de um natimorto ou de um nascido vivo morrer ainda na primeira semana de vida1. Reflete a qualidade da assistência prestada durante o atendimento prénatal, parto, nascimento e atendimento ao recém-nascido, e sofre interferência do contexto demográfico e socioeconômico da população. De modo que este indicador contribui para subsidiar o planejamento, a gestão e a avaliação de políticas públicas de saúde². 
É relevante ressaltar que o período perinatal pode apresentar diferentes definições nos estudos científicos, e este pode estar relacionado à dependência que a sobrevida dos recém-nascidos de extremo baixo peso apresentam, influenciado pelos recursos tecnológicos disponíveis. Como mostra o estudo com dados secundários de um país desenvolvido, calcularam-se as taxas de mortalidade perinatal a partir de duas definições. Contudo, ambas com resultados de um dígito inferior a dez, a saber: a primeira contendo óbitos a partir de 28 semanas até o sétimo dia de vida, identificada a taxa de 6,2 óbitos por 1.000 nascidos vivos; e, para a segunda definição, os óbitos a partir de 20 semanas até 28 dias de vida, que estimou 9,9 óbitos por 1.000 nascidos vivos ${ }^{3}$. As taxas encontradas são inferiores às de países subdesenvolvidos devido à não ocorrência de mortes evitáveis, apenas devidas às causas de mortes irredutíveis, como as anomalias congênitas incompatíveis com a vida 4 .

No Brasil, a definição utilizada para mortalidade perinatal compreende o componente fetal e o neonatal precoce, englobando os óbitos ocorridos a partir da $22^{\text {a }}$ semana de gestação até o sexto dia de vida completo². O período neonatal precoce se relaciona com a mortalidade infantil, que sofreu redução expressiva nos últimos anos. Entretanto, a redução com menor impacto foi para o período neonatal precoce, que passou a representar mais da metade dos óbitos ocorridos em menores de um ano ${ }^{5}$. Este cenário impõe aos stakeholders da área a necessidade de maior conhecimento sobre seus determinantes e das características que cercam este segmento da mortalidade infantil.

Ainda, no Brasil, em 2014, houve 7,5 óbitos neonatais precoces por mil nascidos vivos, com significativa diferença regional, sobretudo entre as regiões Norte $(8,9)$ e Sul $(5,6)$. Apontam-se como principais causas de morte os fatores maternos e perinatais de causas redutiveis como: a prematuridade, os fatores maternos, as infecções perinatais, a asfixia, as afecções respiratórias e as anomalias congênitas ${ }^{5}$. Estas condições, possivelmente, teriam sido evitadas se tivessem sido implementadas as ações para favorecimento da qualidade na assistência ao pré-natal, ao parto, ao nascimento e ao atendimento ao recém-nascido, nos diversos níveis de complexidade de atenção à saúde ${ }^{6}$.

No estado do Paraná, entre 2006 e 2014, a taxa de mortalidade perinatal foi de 14,3 óbitos por mil nascidos vivos, com predominância do componente fetal, com uma redução de $11,7 \%$ no período, gradativa e lenta, sobretudo pela redução de $18,1 \%$ do componente neonatal precoce. Foi observada frente aos investimentos na área da saúde materna e infantil?. Ainda assim, a taxa se apresenta além dos valores internacionais, em especial, apresentados por países desenvolvidos.

Entende-se que investimentos devem ser alocados para o processo de redução da mortalidade perinatal, com base em dados confiáveis, capazes de manifestar a cobertura real das intervenções de saúde; caracterizar o nível de qualidade da assistência prestada; e quantificar os indicadores passíveis de mensuração de desigualdades. Um Sistema de Saúde por meio de seus serviços deve fortalecer sua competência analítica, apoiada estatisticamente para mensurar o impacto das intervenções de saúde e, assim, gerar informações que possam subsidiar a tomada de decisão no desenvolvimento das políticas públicas de saúde voltada para a qualificação de assistênciå.

Entende-se que a diversidade econômica regional precipita fragilidades no acesso aos serviços de saúde, de modo que a meta seja a implementação de ações estratégicas para minimizar as desigualdades sociais, econômicas e demográficas. Nesta perspectiva, o estudo dos óbitos perinatais segundo o local de residência, pode produzir evidências científicas locais para a tomada de decisão nas diferentes esferas de governo pois, em uma dada região de saúde, o local sofre interferência relativa à disponibilidade e à qualidade dos serviços. Destarte, considerando os dispositivos do regime fiscal da Emenda Constitucional $n^{\circ} 95$ de 2016. com um limite para os gastos do governo federal, segundo as políticas públicas de saúde, será possível 
a priorização das regiões para investimentos que contribuam para a transformação social e para a redução de gastos públicos com saúde.

Deste desenvolvimento emergiu a pergunta de pesquisa: Qual a correlação espacial entre a mortalidade perinatal e as condições sociais, econômicas e demográficas dos municípios, no estado do Paraná? Assim, o objetivo desta pesquisa foi investigar a correlação espacial entre a mortalidade perinatal e os indicadores sociais, econômicos e demográficos dos municípios do estado do Paraná, em período anterior à aprovação da Emenda Constitucional $n^{\circ} 95$.

\section{MÉTODO}

Trata-se de uma pesquisa epidemiológica, do tipo ecológica, realizada no Paraná no período de 2006 a 2015, a qual antecede os reflexos da crise econômica enfrentada no Brasil e da aprovação da Emenda Constitucional n 95 de 2016 para contenção de gastos na saúde. O estado é organizado em 399 municípios, com estrutura dos seus serviços de saúde em 22 Regionais de Saúde (RS), que se articulam em quatro macrorregionais de saúde, todas integradas na lógica da Rede de Atenção à Saúde. Este modelo organizacional foi implantado em 2012, ofertando assistência à saúde nos três níveis de atenção em todo o estado $^{10}$, segundo as regiões administrativas apresentadas na Figura 1.

Utilizaram-se para o acesso ao número de óbitos perinatais e nascidos vivos, dados secundários disponibilizados on-line pelo Sistema de Informação sobre Mortalidade e Nascidos Vivos, do Departamento de Informática do Sistema Único de Saúde (DATASUS)ำ. Analisaram-se variáveis sociais, econômicas e demográficas, do Instituto Brasileiro de Geografia e Estatística (IBGE), referentes a 2010, disponíveis na base de dados $B D E w e b^{\circledR}$ do Instituto Paranaense de Desenvolvimento Econômico e Social (IPARDES)12, a saber: taxa de analfabetismo em menores de 15 anos; taxa de atividade com 18 anos ou mais; grau de urbanização: taxa de fecundidade entre 15 a 17 anos; Índice de Gini; e índice de Desenvolvimento Humano Municipal. Estes dados foram coletados e revisados por dupla checagem.

Figura 1. Distribuição das Regionais de Saúde e dos municípios do estado do Paraná, Brasil, 2019.

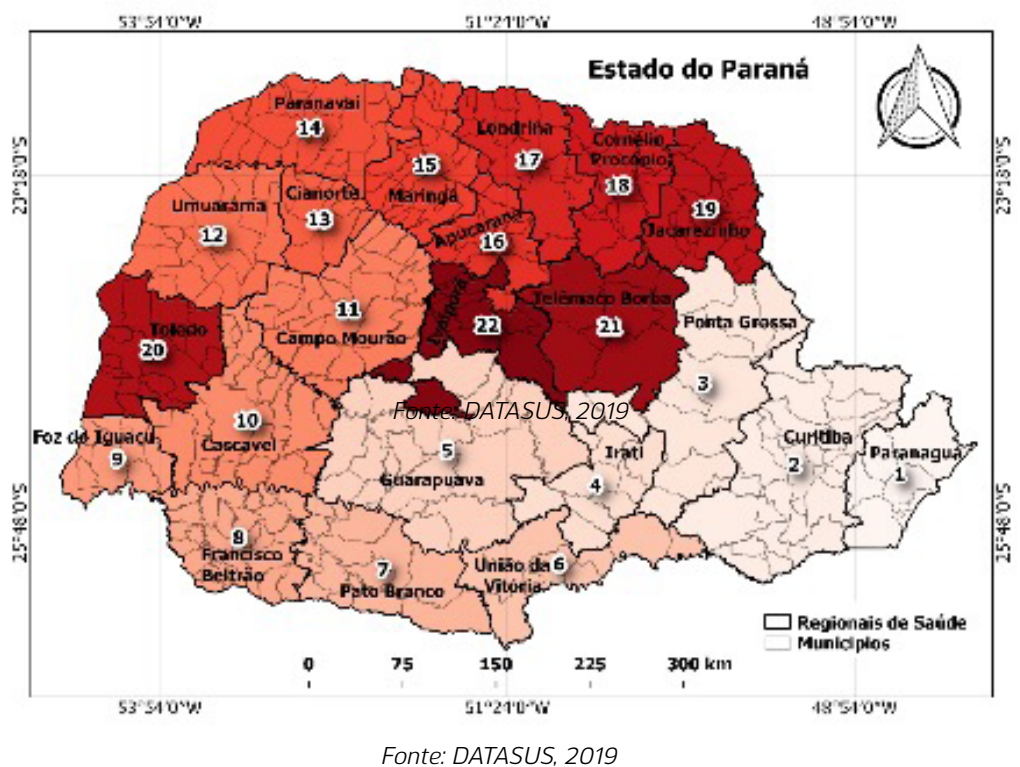


A ferramenta GeoDa na versão 0.9.5-iC foi utilizada para o cálculo da Taxa de Mortalidade Perinatal (TMP) suavizada espacialmente mediante o Spacial Empirical Baye. Esta suavização possibilitou a minimização do viés de variabilidade da taxa, sobretudo pela inclusão de municípios com pequenas populações, considerando no cálculo a soma ponderada da taxa bruta com a média local do evento, pela matriz de pesos de rainha, a fim de evitar falácia ecológica. A TMP consistiu na razão entre os óbitos perinatais (ocorridos entre a $22^{a}$ semana de gestação até o sexto dia completo de vida) e o total de nascidos vivos somados aos óbitos fetais, de uma dada região, em um determinado tempo. A partir desta taxa foi realizada a análise pelo Índice Global de Moran, univariado da TPM e bivariado entre a TMP e as variáveis sociais, econômicas e demográficas.

O checklist Strengthening the Reporting of Observational Studies in Epidemiology foi utilizado desde a concepção dessa pesquisa, como apoio para a estruturação e desenvolvimento, a fim de garantir a produção de informações precisas e completas ${ }^{13}$. Este estudo foi aprovado pelo Comitê de Ética em Pesquisa do Setor de Ciências da Saúde da Universidade Federal do Paraná, sob o parecer nº 362.767.

\section{RESULTADOS}

Na análise univariada do Índice de Moran identificou-se a autocorrelação espacial positiva ou direta $(0,638)$ da TMP, evidenciando a dependência espacial entre municípios e seus vizinhos. A partir desta análise afirma-se, portanto, que municípios com alta TMP fazem vizinhança com outros que também apresentam alta TMP, e ainda, os com baixa TMP fazem vizinhança com municípios que também apresentam baixa TMP (Figura 2).

O Índice Local de Moran favoreceu o detalhamento da correlação espacial, com a identificação dos municípios que formaram conglomerados espaciais, denominados de clusters (Figura 3). Nesta análise, 66 municípios apresentaram correlação espacial direta ou positiva, classificados como cluster alto-alto, ou seja, municípios com alta TMP que eram vizinhos de outros que também apresentavam alta TMP. Estes podem ser visualizados pela composição de três clusters exibidos na cor vermelha-escura: o menor, composto por sete municípios localizados na mesorregião Noroeste abrangendo três RS (12 $13^{\mathrm{a}}$ e 14 $4^{\mathrm{a}}$ ); outro com 23 munícipios no Centro-Sul em quatro RS (4 $4^{\mathrm{a}}, 5^{\mathrm{a}}, 7^{\mathrm{a}}$ e $22^{\mathrm{a}}$ ); e por fim, o maior com 36 municípios localizados no Norte Pioneiro e Centro-Oriental, em cinco RS $\left(2^{\mathrm{a}}, 3^{\mathrm{a}}, 18^{\mathrm{a}}, 19^{\mathrm{a}}\right.$, e $\left.21^{\mathrm{a}}\right)$.

Figura 2 - Diagrama de dispersão da análise univariada da Taxa de Mortalidade Perinatal do Índice Global de Moran, Paraná, Brasil, 2006 a 2015.

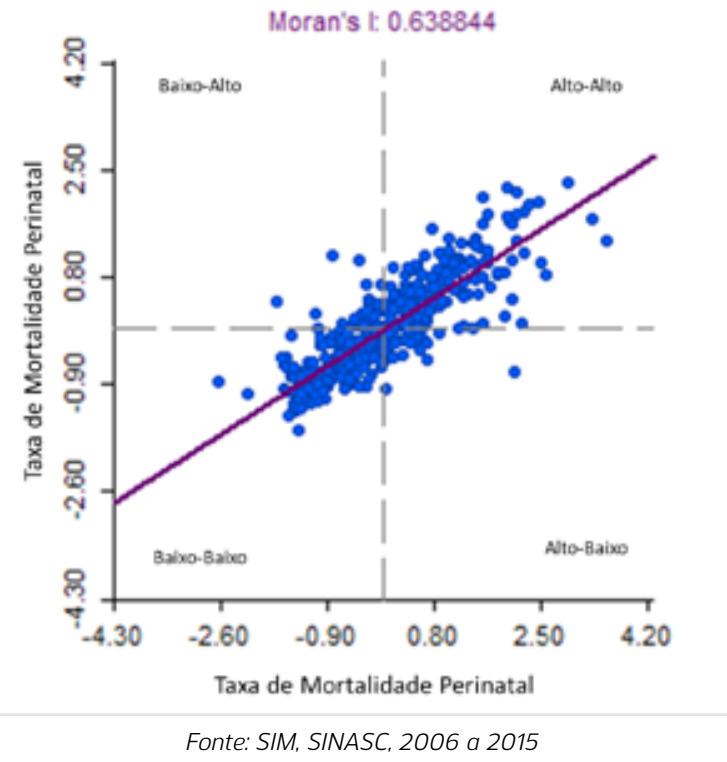


Figura 3 - Distribuição espacial da Taxa de Mortalidade Perinatal, segundo a análise univariada do Índice Local de Moran, Paraná, Brasil, 2006 a 2015

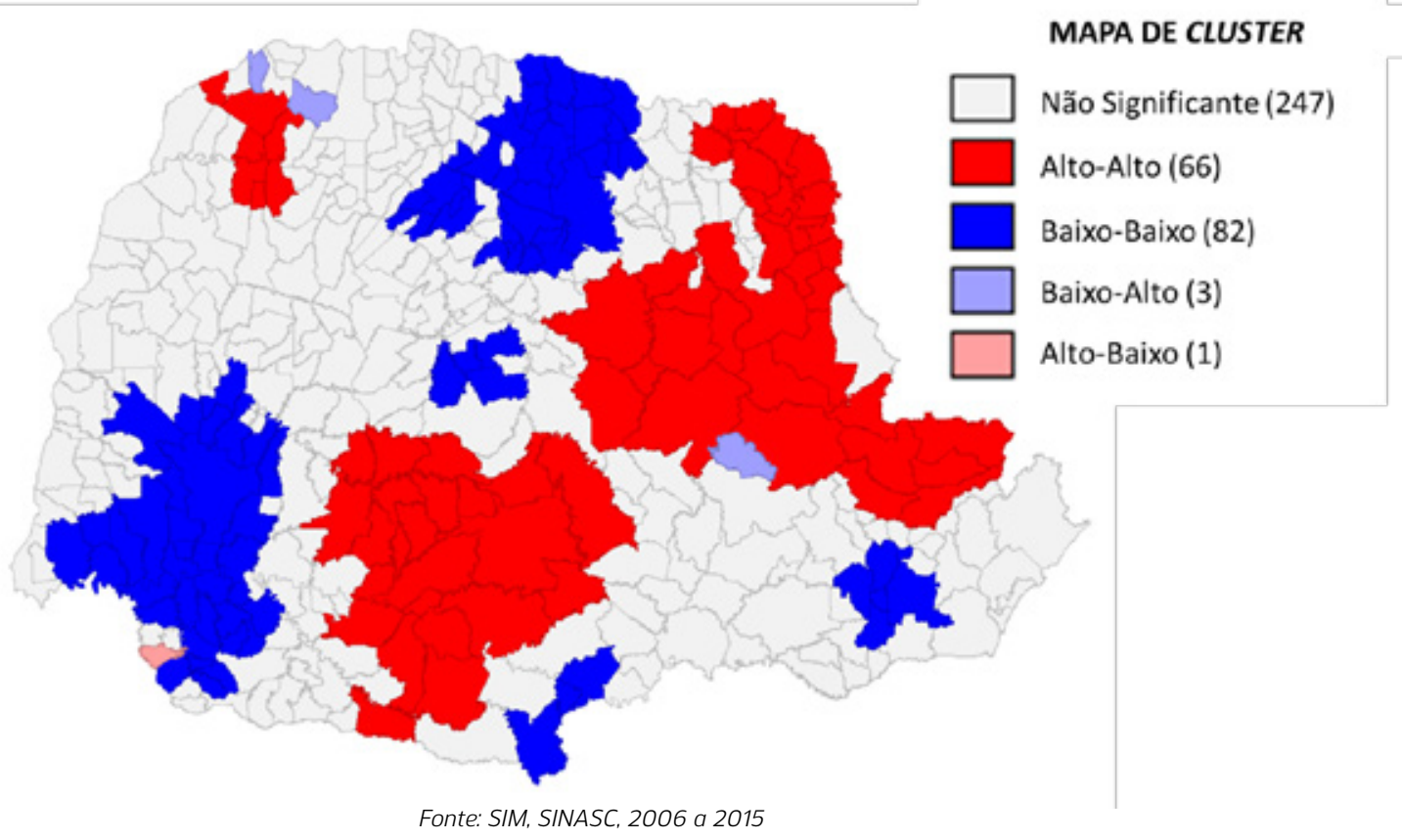

Do mesmo modo, 82 municípios compuseram cinco clusters com correlação espacial direta ou positiva, mas de classificação baixo-baixo, municípios com baixa TMP fazem vizinhança com outros municípios que também apresentam baixa TMP, que foram apresentados na cor azul-escuro (Figura 3). 0 maior, composto por 35 municípios, localizado nas mesorregiões Oeste e Sudeste abrangendo quatro RS ( $8^{\mathrm{a}}, 9^{\mathrm{a}}, 10^{\mathrm{a}}$ e $\left.20^{\mathrm{a}}\right)$; 0 segundo maior com 34 municípios do Norte Central ao Norte Pioneiro, também em quatro RS (15a $16^{\mathrm{a}}, 17^{\mathrm{a}} \mathrm{e}$

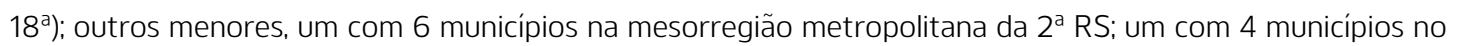
Norte Central da 22 ${ }^{\mathrm{a}} \mathrm{RS}$; e, por fim, com 3 municípios no Sudeste da $6^{\mathrm{a}} \mathrm{RS}$.

Todavia, um município foi classificado como alto-baixo, apresentado na cor vermelho-claro (Figura 3). Portanto, apresenta correlação espacial negativa ou inversa, e é um município com alta TMP que faz vizinhança com municípios que apresentam baixa TMP. Está localizado na mesorregião Sudoeste ( $\left.8^{\text {a }} \mathrm{RS}\right)$. Ao passo que três municípios foram classificados como baixo-alto, e são os municípios que com baixa TMP fazem vizinhança com outros com alta TMP de cor azul-claro (Figura 3). Dos quais, dois estão localizados na mesorregião Noroeste (14 $14^{a}$ RS) e um na Centro-Oriental ( $3^{a}$ RS). Ademais, 247 municípios não apresentaram significância estatística e estão apresentados na coloração branca no mapa (Figura 3).

Além disso, na análise bivariada com o Índice de Moran, foi aplicada para a correlação da TMP às variáveis sociais, econômicas e demográficas dos municípios. Identificou-se correlação direta ou positiva, ou seja, a TMP foi maior em municípios que apresentavam as variáveis a seguir elevadas: taxa de analfabetismo em maiores de 15 anos $(0,183)$, taxa de fecundidade entre 15 a 17 anos $(0,074)$ e Índice de Gini $(0,143)$. Ao contrário, apresentaram correlação inversa ou negativa com a TMP, as variáveis: taxa de atividade em maiores de 18 anos (-0,142), grau de urbanização $(-0,111)$ e Índice de Desenvolvimento Humano Municipal $(-0,276)$. A TMP apresentou-se menor em municípios com estes indicadores elevados (Figura 4). 
Figura 4 - Diagrama de dispersão da análise bivariada da Taxa de Mortalidade Perinatal e do Índice Global de Moran, Paraná, Brasil, 2006 a 2015.
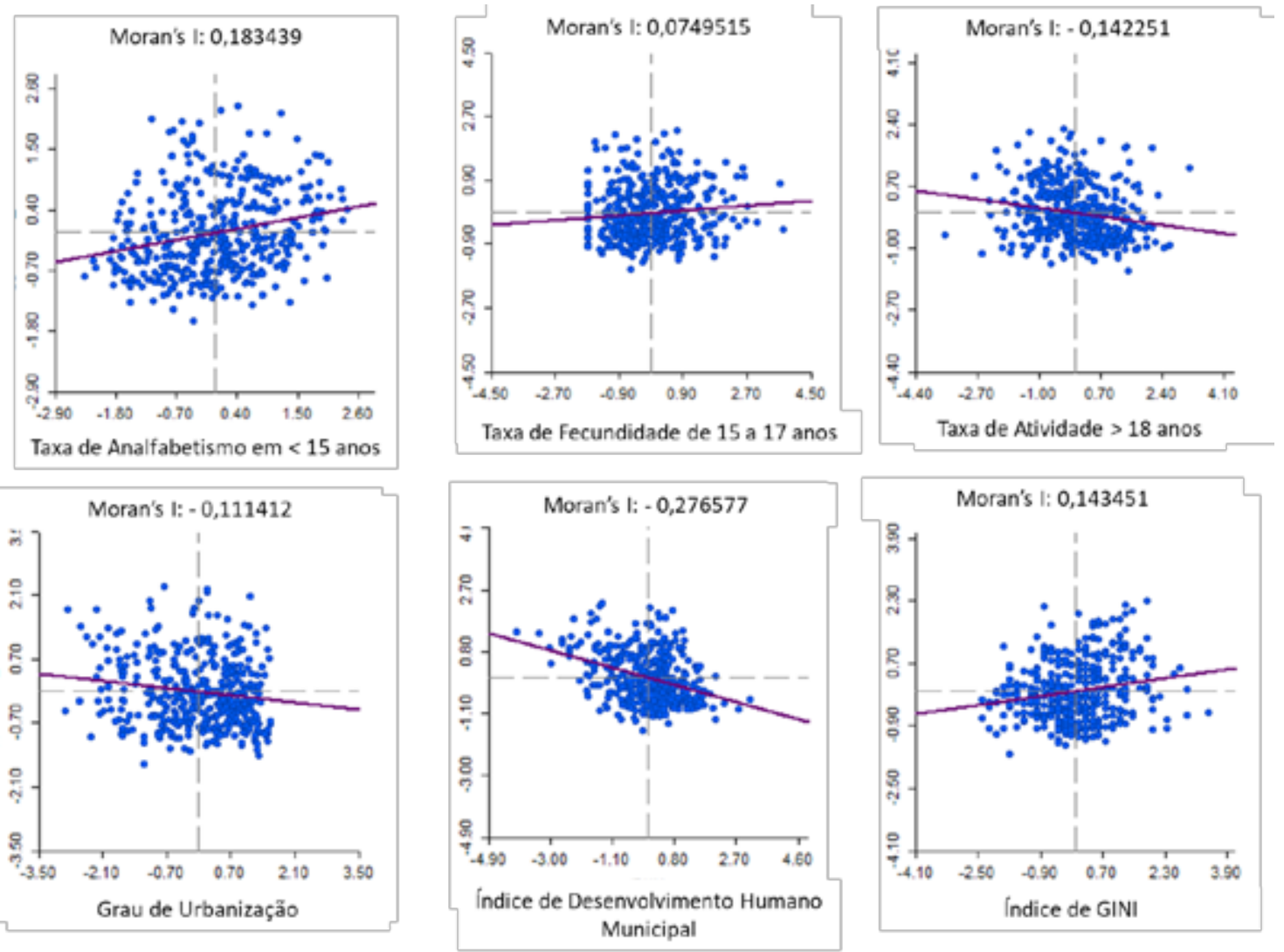

Fonte: SIM, SINASC, 2006 a 2015

\section{DISCUSSÃO}

Não foram identificados na literatura científica estudos que correlacionassem a mortalidade perinatal espacialmente com indicadores sociais, econômicos e demográficos no estado do Paraná. Portanto, vislumbrou-se como favorecedora esta produção se valendo de recurso tecnológico para gerar conhecimento que poderá subsidiar a tomada de decisão na continuidade do desenvolvimento das políticas públicas de saúde materna e infantil. Conhecer a relação do óbito perinatal e o espaço em que ele ocorre a partir de ferramentas da análise espacial permitiu identificar regiões vulneráveis no estado e compreender a relação entre o evento e os municípios que fazem vizinhança entre si. Esta perspectiva pode subsidiar estratégias de qualificação nas condições sociais, econômicas e demográficas das regiões paranaenses.

Em estudo descritivo realizado no período de 1999 a 2010, no Paraná, analisou-se o valor da TMP segundo as Regionais de Saúde constatando-se redução de 28,2\% e concluiu que as RS apresentam diversidades entre si, possivelmente por influência das características dos municípios que as compõem¹4. Este impacto provavelmente está relacionado às características sociais, econômicas e demográficas de cada município, considerando que elas podem convergir com dificuldades de acesso oportuno aos serviços de saúde. 
O estudo citado tem resultado confluente aos da presente pesquisa, tendo em vista que a $5^{\text {a }}$ RS apresentou a maior TMP no estado do Paraná. Os municípios que a compõem, apesar de apresentarem melhora no indicador de mortalidade perinatal, permaneceram ativos na formação de um cluster altoalto. Fato que evidencia a importância do estabelecimento de metas de redução da TMP nesta RS, com o comprometimento participativo entre gestores, profissionais de saúde e comunidade. As metas poderiam ser contempladas pela ampliação ao acesso aos serviços de saúde e com o incremento de maior qualidade ao atendimento pré-natal e ao recém-nascido de risco ${ }^{14}$.

Uma estratégia para a maximização do impacto das iniciativas é a atribuição de recursos econômicos às regiões mais precárias, para o favorecimento da ampliação do acesso aos serviços de saúde, respeitando à diretriz de equidade do Sistema Único de Saúde ${ }^{15}$. Visto que esta necessidade é reiterada com a presente pesquisa ao identificar correlação espacial de elevação da TMP com a elevação da taxa de analfabetismo em maiores de 15 anos, da taxa de fecundidade entre 15 a 17 anos e do Índice de Gini.

A discussão entre a relação da desigualdade social e o seu impacto sobre os resultados de saúde já está estabelecida na literatura, como mostra o estudo ecológico realizado no Rio de Janeiro. 0 Índice de Gini, ao apresentar o grau de concentração de renda, evidencia as condições de privação social e material que contribuem com a redução da qualidade de vida de uma população. A pobreza provoca condições que levam à ruptura familiar e social, gerando forte frustração e estresse, e está concentrada espacialmente de modo que qualquer fenômeno relacionado a ela, como a mortalidade perinatal, também o serán 16 .

A análise da mortalidade infantil em países do continente europeu e na China identificou o mesmo comportamento de redução da TMP. Apontou que a expectativa de vida da criança doente vem aumentando devido ao desenvolvimento social e econômico destas regiões ${ }^{17}$. Fato que reforça a necessidade de implantação de estratégias de promoção à saúde para a redução da desigualdade social, não exclusivamente no âmbito da área da saúde, mas contemplando todas as dimensões das políticas públicas sociais.

Confirma-se que os municípios que apresentaram nesta pesquisa as menores TMP mantinham correlação inversa com as variáveis socioeconômicas, como: Índice de Desenvolvimento Humano Municipal, grau de urbanização e taxa de atividade de maiores de 18 anos. Uma pesquisa internacional relacionou a redução da TMP com maiores investimentos na área da saúde voltados para a capacitação profissional, além da disponibilização de recursos tecnológicos e o planejamento dos partos de riscos em hospitais de referência ${ }^{18}$

Estudo com análise espacial confirma estes achados, apontando a distribuição da mortalidade perinatal de forma heterogênea no espaço, além de ser incrementada pela menor escolaridade materna18. Estudo ecológico nacional também confirmou a correlação da mortalidade neonatal com os fatores sociais e econômicos nos estados brasileiros. Apontou a renda domiciliar per capita maior nos estados da região Sul, Sudeste e parte do Centro-Oeste, quando comparada aos estados do Norte e Nordeste, bem como a formação de cluster alto-alto no Nordeste, sugerindo correlação espacial entre a mortalidade neonatal devido à baixa renda domiciliar per capita ${ }^{20}$.

Outro estudo, do tipo ecológico, analisou a correlação espacial entre os fatores de risco à mortalidade neonatal também utilizando o Índice de Moran. Este identificou correlação direta, com maior mortalidade entre as mães adolescentes, com baixa escolaridade, que realizaram número de consultas de pré-natal abaixo do recomendado e os hospitais públicos constituíram-se o local de nascimento ${ }^{21}$. Condições possivelmente relacionadas às contingências sociais e econômicas do município estudado. Estes achados corroboram com evidência da forte relação da TMP com as variáveis sociais e econômicas dos municípios, possivelmente devido a afetar o acesso da população a serviços de qualidade e, consequentemente, ao atendimento oportuno no pré-natal e no parto. 
A pobreza apresenta importante determinação social sobre a mortalidade neonatal, justificando a consideração do cenário econômico sobre este resultado de saúde, devido à interferência no acesso à renda familiar. Destaca-se que o cenário de redução da mortalidade apresentou desaceleração devido à crise econômica enfrentada pelo Brasil desde 2014, como mostra um estudo de microssimulação, realizado em âmbito nacional. As políticas de austeridade aplicadas para o enfrentamento da crise, caracterizadas pela redução de investimentos no Programa Bolsa Família e na Estratégia Saúde da Família, após a aprovação da Emenda Constitucional $n^{\circ} 95$ de 2016, alteram o estado de bem-estar social e favorecem a morbimortalidade de crianças $^{22}$.

Na Grécia, entre 2004 e 2016, a análise da mortalidade infantil durante a crise econômica de 2008, com a redução do Produto Interno Bruto (-26\%) e dos investimentos em saúde (-34\%), identificou aumento dos óbitos a partir de 2012. Quatro anos após o início da crise evidenciou-se impacto negativo na mortalidade neonatal. Aponta-se maior probabilidade de óbitos de filhos de mulheres residentes em áreas rurais devido à presença de barreiras geográficas, e por apresentarem menor escolaridade, menor renda, gravidez em idade mais jovem e maior número de filhos, quando comparadas às mulheres residentes em área urbana. Foram sugeridas melhorias quanto à qualidade da assistência perinatal com referência ao local de parto, implantação de práticas baseadas em evidência, bem como aumento nos investimentos para a contratação de profissionais da saúde ${ }^{23}$.

A principal limitação deste estudo foi a ausência de base de dados públicos com variáveis que refletissem o acesso aos serviços de saúde e à qualidade da assistência à saúde materna e infantil prestada em cada município, para aprofundamento da compreensão do óbito perinatal. Fato que reflete na impossibilidade de relacionar o impacto da implantação da Rede de Atenção à Saúde Materna e Infantil, em 2012, passível de mensuração entre sete a dez anos após a implantação ${ }^{24}$. Este programa, busca favorecer o acesso aos serviços de saúde pela implementação da estratificação de risco da gestante estabelecida durante todo o processo de atendimento pré-natal, bem como, do recém-nascido. Acredita-se no grande potencial que possui esta estratificação para o favorecimento da continuidade da redução da Mortalidade Perinatal no Estado 25 .

\section{CONCLUSÃO}

A análise espacial se mostrou uma ferramenta tecnológica valiosa para o monitoramento da mortalidade perinatal no estado do Paraná e pode ser aplicada a outros cenários. Este recurso analítico permitiu a identificação geográfica de locais que necessitam de alocação de investimentos na saúde materna e infantil, com o propósito de contribuir com a política pública vigente. Mediante os achados se recomendam investimentos direcionados para a implementação de ações específicas e oportunas que minimizem a ocorrência do óbito perinatal, de acordo com as variáveis apontadas. Esta análise confirma o impacto das condições socioeconômicas negativas dos municípios nos indicadores de saúde, para o qual sugere-se a intersecção do trabalho da saúde com as demais políticas públicas, em assentimento com as recomendações dos Objetivos do Desenvolvimento Sustentável.

Por fim, a educação formal de uma população e a igualdade de direitos e oportunidades atua significativamente sobre a redução da TMP. Converge com as menores taxas a vigência de melhor desenvolvimento humano, urbanização e possibilidade de trabalho para os maiores de 18 anos de idade. Destaca-se que o desenvolvimento de novas pesquisas que avaliem o processo, a estrutura e os resultados 
da assistência oferecida por serviços de saúde da Rede de Atenção à Saúde Materna e Infantil do estado do Paraná, favorecerão a continuidade da redução da Mortalidade Perinatal.

\section{AGRADECIMENTOS}

O presente trabalho foi realizado com apoio da Coordenação de Aperfeiçoamento de Pessoal de Nível Superior - Brasil (CAPES) - Código de Financiamento 001.

\section{REFERÊNCIAS}

1. Brasil (Ministério da Saúde). Sintese de evidências para políticas de saúde Mortalidade perinatal. Secretaria de Ciência, Tecnologia e Insumos Estratégicos, Departamento de Ciência e Tecnologia. 2. ed. Braślia: Ministério da Saúde. 2016.

2. Organização Mundial da Saúde. CID 10 / Organização Mundial de Saúde: tradução Centro Colaborador da OMS para Classificação em Português. 10. ed. Rev. - São Paulo: Editora da Universidade de São Paulo. 2007.

3. MacDorman MF, Gregory ECW. Fetal and Perinatal Mortality: United States, 2013. Natl Vital Stat Rep. [Internet].2015 [cited Abr 10, 2017];64(8):1-24. Disponivel em: http://europepmc.org/abstract/med/26222771.

4. World Health Organization. Neonatal and perinatal mortality 2004. Country, regional and global estimates. Geneva: WHO; 2007. 20p Disponivel em: http://www.who.int/maternal_child_adolescent/documents/9789241596145/en.

5. Brasil. Ministério da Saúde. Secretaria de Vigilância em Saúde. Saúde Brasil 2015/2016: uma análise da situação de saúde e da epidemia pelo vírus Zika e por outras doenças transmitidas pelo Aedes Aegypti. Brasília: Ministério da Saúde. 2016.

6. Miranda MHH, Fernandes FECV, Campos MEAL. Determinants associated to perinatal mortality and associated factors. Rev Enferm. UFPE. 2017:11(3):1171-8. DOI: 10.5205/reuol.10544-93905-1-RV.1103201707.

7. Migoto MT. Oliveira RP. Freire MHS. Análise da Mortalidade Perinatal e seus fatores associados. Rev Baiana Enferm. 2018:32:e26249. DOI: 10.18471/rbe.v32.26249

8. Boerma T. Collaboration. Countdown to 2030: tracking progress towards universal coverage for reproductive, maternal, newborn, and child health. Lancet. 2018:30:1-11. DOI: 10.1016/S0140-6736(18)30104-1.

9. Brasil. Casa Civil. Emenda Constitucional n 95 de 2016. Disponivel em: http://www.planalto.gov.br/ccivil_03/constituicao/emendas/emc/ emc95.htm

10. Paraná. Secretaria do Estado de Saúde do Paraná. Linha Guia: Rede Mãe Paranaense. [Internet]. 2012 [cited Abr 20, 2017]. Disponivel em: http://www.saude.pr.gov.br/arquivos/File/Master_Governo_LinhaGuia_v3.pdf.

11. Datasus. Informações de Saúde (TABNET). Estatisticas Vitais. [Internet]. 2017 [cited Abr 20, 2017]. Disponivel em: http://www2.datasus. gov.br/DATASUS/index.php?area=0205

12. Ipardes. Instituto Paranaense de Desenvolvimento Econômico e Social. Base de Dados do Estado. [Internet]. 2017 [cited Abr 20. 2017]. Disponivel em: http://www.ipardes.pr.gov.br/anuario_2016/index.html

13. Malta M, Cardoso LO, Bastos FI, Magnanini MMF. Silva CMFP. Iniciativa STROBE: initiative: guidelines on reporting observational studies. Rev Saúde Pública. 2010:44(3):559-65. DOI: 10.1590/S0034-89102010000300021.

14. Vieira TMM, Oliveira RR, Mazza VA, Mathias TAF. Perinatal Mortality and regional diferences in the state of Paraná, Brazil. Cogitare Enferm. 2015; 2(4):783-791. DOI: 10.5380/ce.v20i4.42626.

15. Antunes JLF. Socioeconomic status and health: a discussion of two paradigms. Rev. Saúde Pública. 2008:42(3). DOI: 10.1590/S003489102008005000017

16. Szwarcwald CL, Bastos FI, Esteves MAP, Andrade CLT, Paez MS, Medici EV et al. Desigualdade de renda e situação de saúde: o caso do Rio de Janeiro. Cad Saúde Pública. 1999:15(1):15-28. Disponivel em: https://www.scielosp.org/pdf/csp/1999.v15n1/15-28/pt. 
17. Xu Y, Zhang W, Yang R, Zou C, Zhao Z. Infant mortality and life expectancy in China. Med Sci Monit. 2014;20:379-385. DOI: 10.12659/ MSM.890204.

18. Fuster V. Biodemographic analysis of factors related of perinatal mortality in Portugal (1988-2011). Int J Pediatr. 2016;2016(6123065):10. DOI: 10.1155/2016/6123065

19. Martins EF, Rezende EM, Almeida MCM, Lana FCF. Perinatal Mortality and socio-spatial inequities. Rev Latino-Am Enferm. 2013;21(5):106270. DOI: 10.1590/S0104-11692013000500008.

20. Oliveira GS, Lima MCBM, Lyra CO, Oliveira AGRC. Ferreira MAF. The spatial inequality of neonatal mortality in Brasil: 2006 a 2010. Cien Saude Colet. 2013;18(8):2431-2441. DOI: http://dx.doi.org/10.1590/S1413-81232013000800028.

21. Gonçalves AC, Costa MCN, Braga JU. Análise da distribuição espacial da mortalidade neonatal e dos fatores associados, em Salvador, Bahia, Brasil, no período de 2000 a 2006. Cad Saúde Pública. 2011;27(8):1581-1592. DOI: 10.1590/S0102-311X2011000800013.

22. Rasella D, Basu S, Hone T, Paes-Sousa R, Ocké-Reis CO, Millett C. Child morbidity and mortality associated with alternative policy responses to the economic crisis in Brazil: A nationwide microsimulation study. PLOS Med. 15(5): e1002570. DOI: 10.1371/journal.pmed.1002570.

23. Siahanidou T, Dessypris N, Analitis A, Mihas C, Evangelou E, Chrousos G. Et al. Disparities of infant and neonatal mortality trends in Greece during the years of economic crisis by ethnicity, place of residence and human development index: a nationwide population study. BMJ Open. 2019:9:e025287. DOI: 10.1136/bmjopen-2018-025287.

24. Kellog-Foundation KW. Using a logic models to bring together planning, evaluation and action. Logic model develop guide. Michigan. 2004.

25. Migoto MT, Oliveira RP. Silva AMR, Freire MHS. Mortalidade neonatal precoce e fatores de risco: estudo caso-controle no Paraná. Rev Bras Enferm. 2018;71(5):2675-83. DOI: 10.1590/0034-7167-2016-0586. 